\title{
Evaluación y monitorización de la durabilidad de las cubiertas del Hipódromo de la Zarzuela de Madrid
}

\author{
Assessment and monitoring of durability of shell structures \\ in "Zarzuela Racecourse" Madrid
}

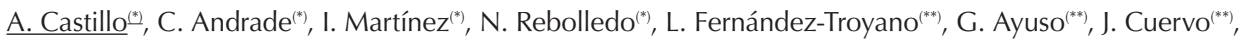
J. Junquera ${ }^{(* *)}$, C. Santana ${ }^{(* *)}$, J. Delgado ${ }^{(* * *)}$

RESUMEN

En el presente trabajo se describen algunos aspectos que se han tenido en cuenta en la restauración que se ha llevado a cabo recientemente en la emblemática obra de Torroja del Hipódromo de La Zarzuela,. Aunque la estructura presenta un muy buen comportamiento, se han producido algunos deterioros locales debido a la corrosión de la armadura por rotura de la impermeabilización y la carbonatacion del hormigón. Lo primero que se realizó fue una evaluación del grado de la corrosión, para posteriormente acometer el proyecto de reparación, que consistió en la inyección de grietas y en el parcheo de zonas deterioradas. Durante la reparación se procedió a la instalación de un sistema de registro de corrosión con sensores permanentes. Se presentan los resultados de la aplicación de técnicas electroquímicas no destructivas utilizadas en la evaluación de estructuras, y se describen el sistema de sensores instalados y sus primeros resultados.

$689-19$

Palabras clave: Hormigón, durabilidad, monitorización, sensores, corrosión.

\section{SUMMARY}

Present paper describes some aspects of the restoration project recently undertaken in La Zarzuela Racecourse, designed by E. Torroja, Despite the good mechanical work of steel, it has started a deterioration process which has caused the reinforcement corrosion by carbonation. It has been detected corrosion attack in some places due the breaking of the upper waterproofing membrane of the shell and concrete carbonation. For the repair first an assessment of the degree of corrosion was made. The restoration project finally consisted in the injection of all the cracks in order to assure the joint work of the shell and the patching of the deteriorated zones. Profiting these works it was installed a monitoring system of corrosion sensors. We present the results of the application of electrochemical techniques, qualitative and quantitative measures used for assessing the residual service life of concrete structures and monitoring, where real Zarzuela Racecourse

Keywords: Concrete, durability, monitoring, sensors, corrosion.

\footnotetext{
${ }^{(*)}$ CISDEM. (CSIC). Madrid, (España)

${ }^{(* *)}$ Carlos Fernández Casado S.L., Madrid, (España)

${ }^{(* * *)}$ J. Junquera, C. Santana Estudio Jerónimo Junquera, S.L., Madrid, (España)

${ }^{(* * *)}$ DRAGADOS, S.A., Madrid, (España)
} 
1. Fotografía panorámica (fotografía del archivo del Instituto Eduardo Torroja) y esquema de construcción (3)

\section{INTRODUCCION}

La construcción del hipódromo de la Zarzuela, declarado patrimonio histórico nacional, fue iniciada su construcción en Madrid en el año 1935, y es una obra del Ingeniero Eduardo Torroja y los arquitectos Arniches y Domínguez. En la actualidad el Hipódromo de la Zarzuela son propiedad del Estado y por tanto son Patrimonio Nacional. El Recinto de Carreras, el "Depósito de la copa", las Ilamadas "Cuadras de Torroja" y las pistas de carreras, están declaradas Bien de Interés Cultural, con categoría de Monumento.Las tres cubiertas de su estructura son consideradas un arte desde el punto de vista ingenieril debido a su perfecto y genial equilibrio físico y mecánico. Se plantearon en su concepción finas láminas de hormigón armado con forma de hiperboloide con espesor variable entre $65 \mathrm{~cm}$ en la zona de pilares y $6 \mathrm{~cm}$ en los bordes. Su sección ligeramente curva en forma de $\mathrm{V}$, apoyada en voladizo de hasta $13 \mathrm{~m}$ de altura sobre un único pilar (Figura 1 ), es posible gracias al inteligente diseño de armados entrelazados y a la instalación de tirantes pasivos de acero situados en su parte trasera. Descripciones detalladas del proyecto y construcción pueden encontrarse en (1), (2) y (3).
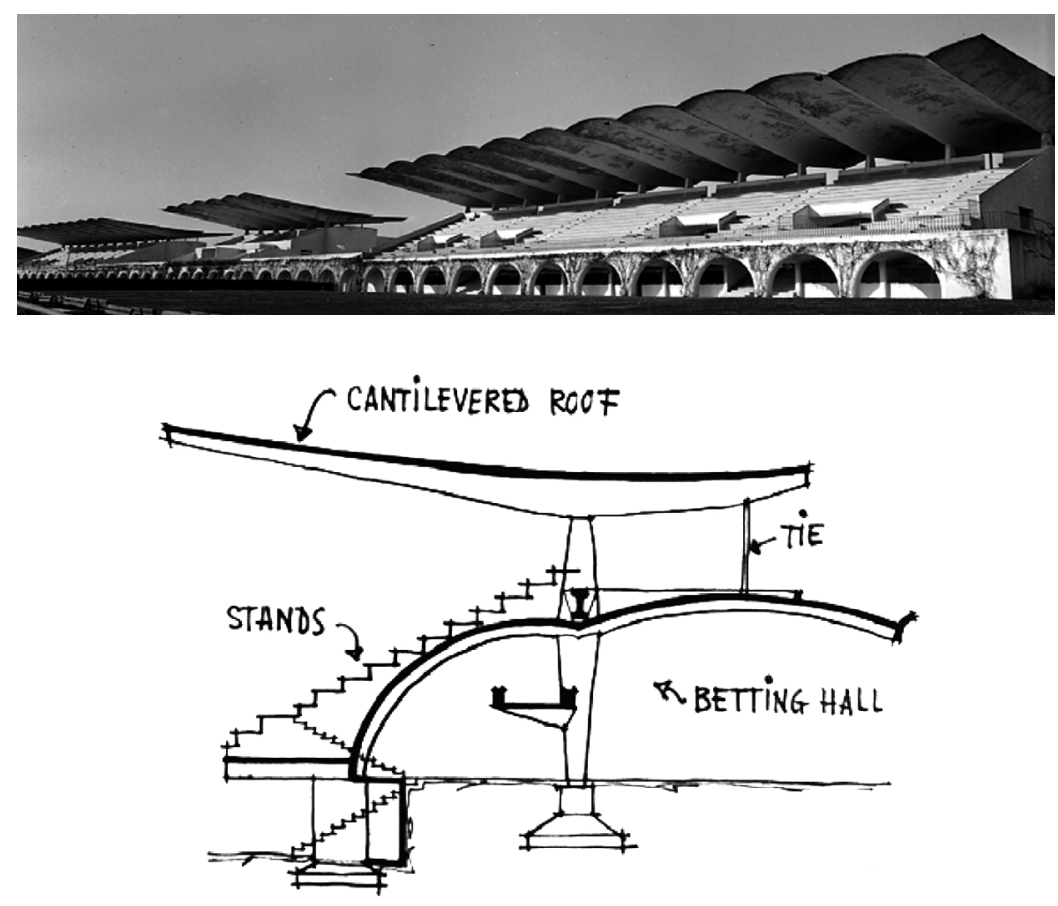

El hipódromo tiene tres tribunas sobre las cuales están dispuestas las cubiertas que se denominan de la siguiente forma:

- Tribuna Norte (C.N.)

- Tribuna Central (C.C.)

- Tribuna Sur (C.S.)
Desde el punto de vista de la durabilidad el comportamiento se puede considerar como muy bueno dado que los deterioros encontrados han sido muy locales y debido a la perdida de la impermeabilización superior de la cubierta que permitió al agua de la lluvia permear a través de la sección de la cubierta. La consideración del grado de buen comportamiento durable se emite al comparar con otras estructuras de hormigón armado menos arriesgadas en su diseño y aparentemente más robustas que han tenido que ser muy reparadas o incluso demolidas con vidas inferiores. El hormigón utilizado, típico de la época y por ello bastante poroso al tener bajo contenido en cemento, estaba parcialmente carbonatado y por ello era necesario plantearse una reparación integral.

La explotación de la estructura como hipódromo ha sufrido diversos avatares por lo que estuvo cerrado al final del siglo XX durante alrededor de una década. Más recientemente se decidió por fin su reapertura y dada la importancia de la obra desde el punto de vista artístico, histórico y de seguridad, las autoridades correspondientes decidieron realizar una obra de restauración de las cubiertas y otras zonas. Aprovechando las inspecciones efectuadas con los proyectistas de la restauración para el dictamen del grado de corrosión de las cubiertas, se les planteó la instalación de un sistema de monitorización mediante sensores (4) y (5), capaces de indicar cualitativamente el riesgo de corrosión en la armadura embebida de la estructura. De este modo, es posible controlar el comportamiento del material frente a la interacción con la acción atmosférica y preveer la posible necesidad de intervenciones de mantenimiento futuras, evitando el avance del deterioro con rapidez y garantías.

En el presente trabajo se describe someramente la evaluación del grado de corrosión efectuada mediante técnicas electroquímicas no-destructivas (6), (7) y (8), que consisten en medidas cuantitativas puntuales de velocidad de corrosión del acero para poder evaluar el elemento en su conjunto y estudiar su evolución después de la reparación. Igualmente se describen los sensores instalados y los primeros datos registrados.

\section{ESTUDIO DEL GRADO DE DETERIORO}

Las cubiertas del Hipódromo de la Zarzuela son estructuras que durante toda su vida en servicio no se ha encontrado documentación que indique que hayan tenido unas actuaciones importantes de mantenimiento. En su parte inferior presentaban zonas reparadas por parcheo y diversas capas de pintura aplicadas, desaparecidas en ciertas 
zonas bien visibles en fotos de principios del siglo XXI. Estos parcheos que presentaban, en algunos casos, desprendimientos y pérdidas de material procedían probablemente de reparaciones puntuales anteriores, siendo algunos de ellos tal vez motivados por la caída de proyectiles durante la guerra civil española (Figura 2-c y d).

En la parte superior de las cubiertas se había colocado un sistema de impermeabilización formado por una imprimación asfáltica sobre la que se dispuso una lámina de aluminio gofrado cuyo estado en 2004 se puede apreciar en Figura 2-a pero que en 2008 esta impermeabilización se encontró muy deteriorada como lo muestra la Figura 2-b. Esta lámina de aluminio (Figura 2-b) se presupone que fue instalada en los años sesenta, posteriormente a la construcción de las cubiertas. El paso del tiempo y la posible falta de mantenimiento han provocado que zonas de esta impermeabilización estuvieran deterioradas y que la imprimación estuviera meteorizada por el efecto de la radiación solar (Figura 2-a)). Además la mayoría de los sumideros de las cubiertas estaban obstruidos, provocando la acumulación de agua estancada, que con el deterioro de la impermeabilización inducía la penetración del agua a través del hormigón. Todo ello originó que se iniciaran procesos de deterioro, entre los cuales se destaca como principal patología la corrosión de su armadura embebida dada la carbonatación del recubrimiento de hormigón.

La carbonatación del hormigón ha sido estudiada y cuantificada in-situ mediante la determinación del frente de carbonatación a través de ensayos colorimétricos. Dichos ensayos consisten en aplicar el indicador fenolftaleína en disolución alcohólica (9) sobre catas abiertas y sobre fractura fresca de muestras en zonas de estudio de las diferentes cubiertas.

Las profundidades del frente de carbonatación de las diferentes muestras extraídas de la superficie inferior de las cubiertas reflejan cómo este ha llegado a la armadura. Conviene resaltar además que los recubrimientos de las armaduras son en general escasos. Por ello, en zonas con penetraciones superiores a 60 $\mathrm{mm}$, el frente llega a alcanzar la segunda capa de armado generando la corrosión generalizada de las armaduras, y llegando a generar pérdidas de sección locales importantes en algunas armaduras. (Figura 3)

Por el contrario, en la superficie superior de las cubiertas no se aprecia frente de carbonatación ya que, aunque la imprimación de impermeabilización estaba deteriorada, en estas zonas hubo encharcamientos de agua durante mucho tiempo, y por ello gran parte de la superficie del hormigón ha estado casi saturada, lo que ralentiza el avance del agresivo por esa superficie. El problema surge cuando el frente alcanza las armaduras por la parte de la superficie inferior de la cubierta, y se acelera la corrosión existente debido al alto grado de contenido de agua en el interior del hormigón.

Otra observación realizada que no ha provocado corrosión pero que resultó relevante es la fisuración generalizada que es visible y cuya causa es probablemente la retracción. En el proyecto de rehabilitación se decidió su reparación mediante sellado con el fin de asegurar el comportamiento solidario de todo el material.

También es necesario nombrar los tirantes que soportan las cubiertas desde su parte
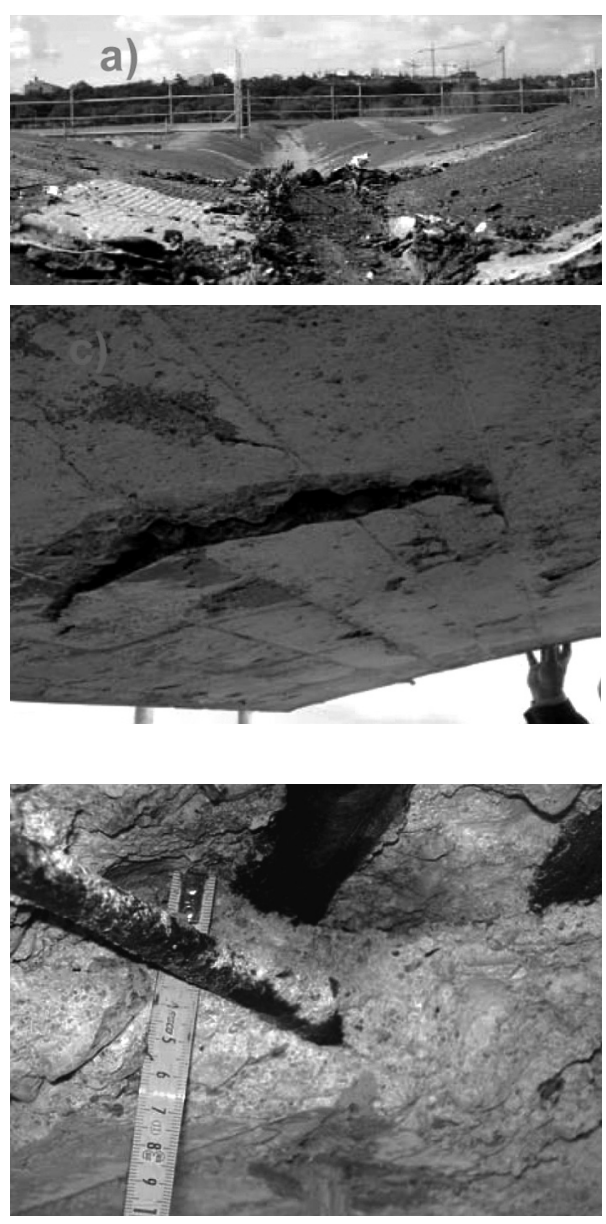

posterior, en los que se tomaron muestras de la Cubierta Norte. Estos tirantes estaban compuestos por tres cordones de acero lisos recubiertos por mortero y todo ello a su vez recubierto por una envoltura de fibrocemento que realizaba la función de impermeabilización y de encofrado perdido. (Figura 4)

De los tirantes se tomaron muestras de mortero para comprobar el posible frente
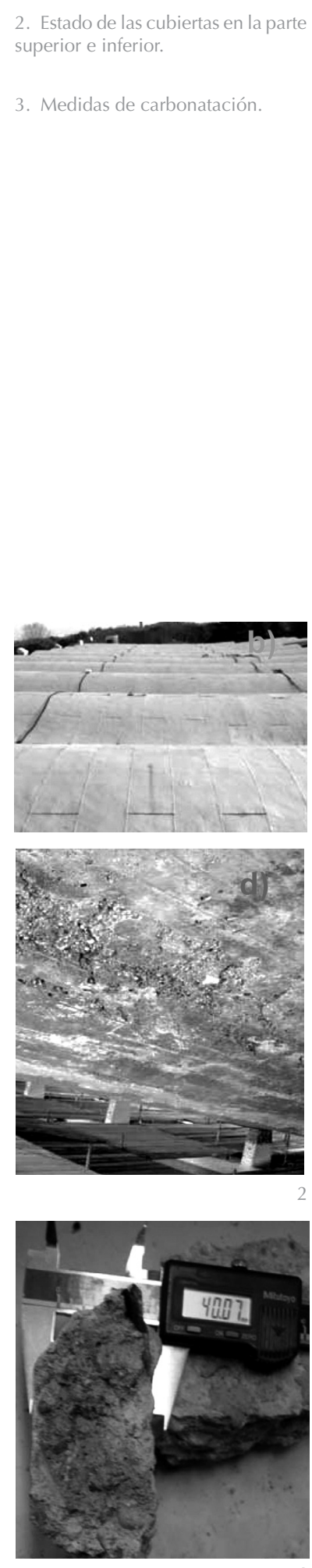


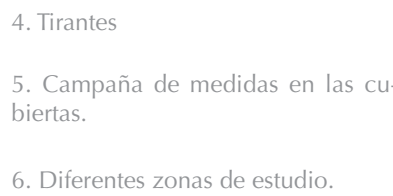

6. Diferentes zonas de estudio.

de carbonatación que resultó inexistente posiblemente por la protección que ha supuesto el recubrimiento de fibrocemento. La ausencia de carbonatación justifica el casi perfecto estado en el que se encontraron los cordones de acero.

\section{EVALUACIÓN DE LA CORROSIÓN}

Después de limpiar la pintura de la superficie inferior de las cubiertas y la capa de
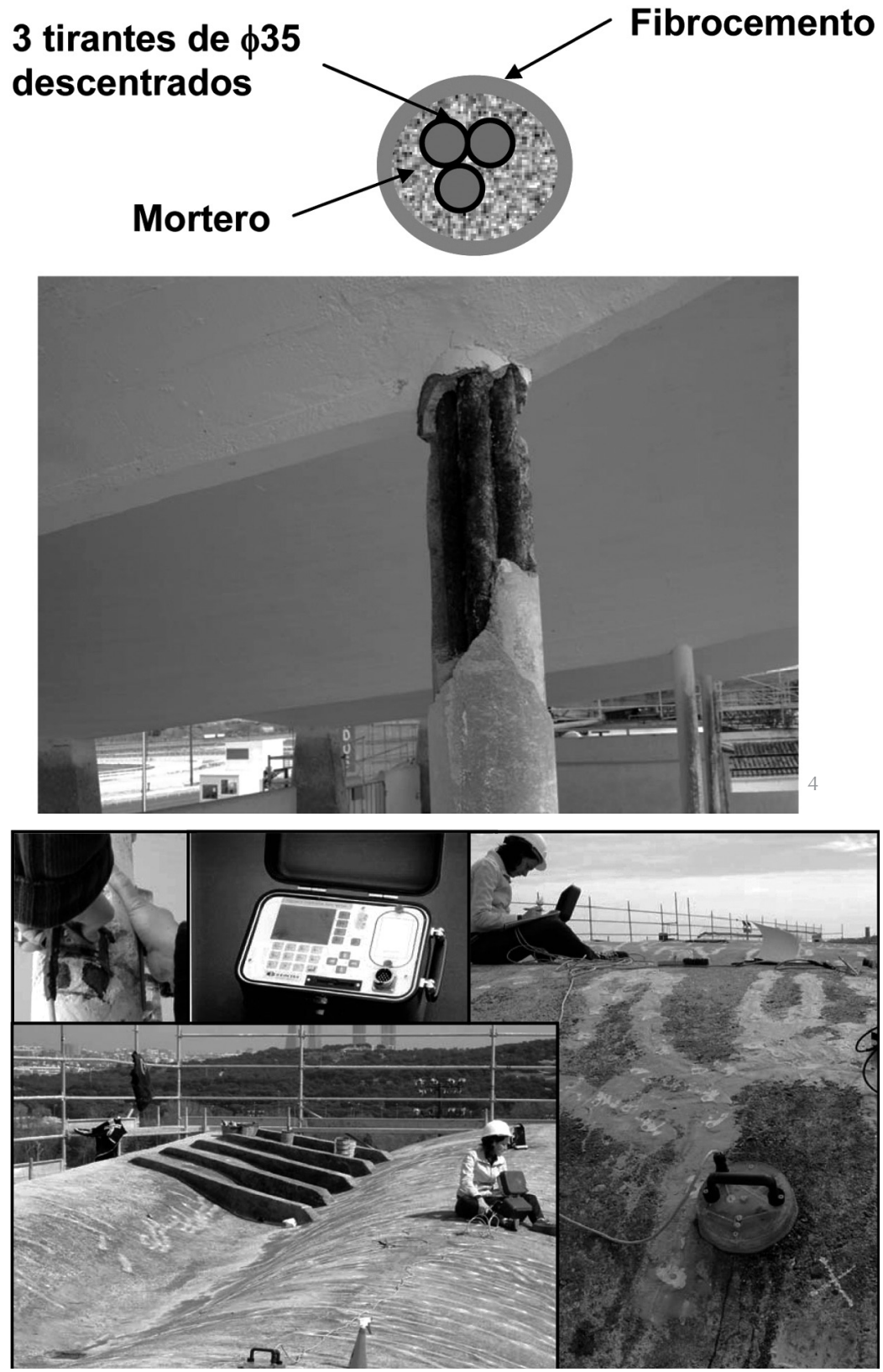

impermeabilización de la superficie superior con agua a presión, se realizó una evaluación del grado de corrosión existente mediante inspección visual y aplicando métodos electroquímicos no destructivos basados en la técnica de la Resistencia a la Polarización para verificar la velocidad de corrosión. (Figura 5)
La velocidad de corrosión (evaluada por el método de confinamiento modulado (6) de la corriente con el corrosímetro portátil Gecor 08) se cuantificó en diferentes áreas de las tres cubiertas (Figura 6). Como se puede ver en la Figura 7, la mayoría de los valores de $I_{\text {corr }}$ registrados en la Cubierta Norte se encuentran en el rango entre 0,5 y $1 \mu \mathrm{A} / \mathrm{cm}^{2}$, lo que supone velocidades de corrosión moderadas (10).

Otros indicadores de corrosión, como el potencial de corrosión $\left(\mathrm{E}_{\text {corr }}\right.$ ) y la resistividad eléctrica ( $r)$, también fueron evaluados (Figura 8 y Figura 9). $\mathrm{E}_{\text {corr }}$ se midió con
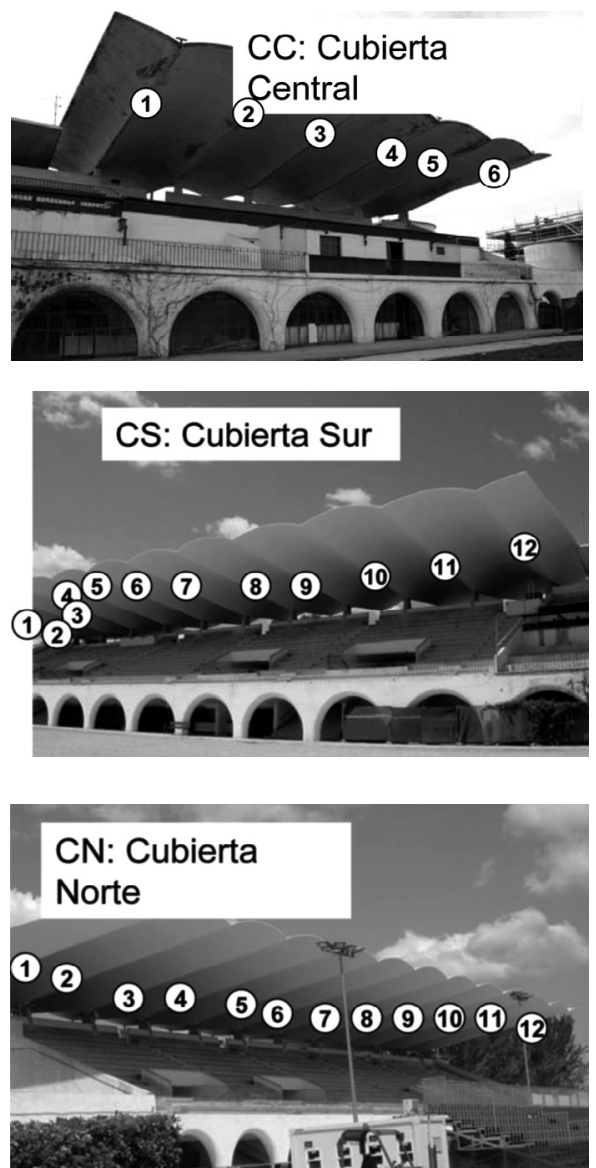

un electrodo de referencia $\mathrm{Cu} / \mathrm{CuSO}_{4}$. La mayoría de los valores medidos estuvieron en el rango entre -250 y $-350 \mathrm{mV}$, lo que significa un riesgo de corrosión intermedio (8). Con respecto a la resistividad, los valores son muy elevados (superiores a $200 \mathrm{~K} \Omega . \mathrm{cm}$ ). Estos altos valores no se corresponden con la porosidad del hormigón, pero podrían ser debidos a la carbonatación del recubrimiento que no siempre permite un buen contacto electrolítico entre el sensor y el hormigón.

La corrosión detectada es generalizada (Figura 10) con pérdida de sección desde despreciable a alrededor de un $10 \%$ Por lo 

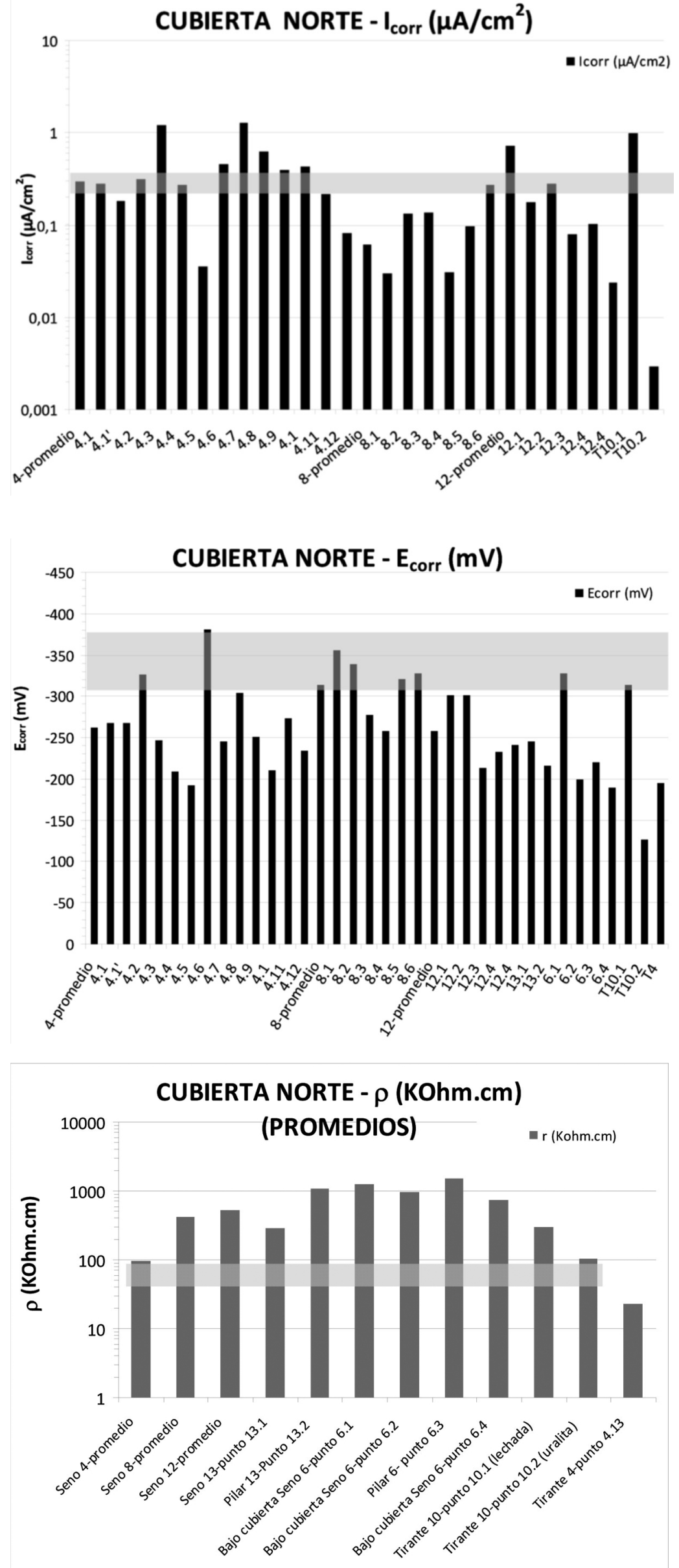


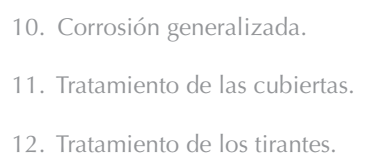

12. Tratamiento de los tirantes. que el grado de corrosión o corrosión acumulada es variable. Esta corrosión es mucho mayor en las zonas que habían perdido la impermeabilización superior donde la pérdida de sección se considero elevada (hasta el $40 \%$ ) si bien estos casos son puntuales y no afectan al comportamiento global de las cubiertas. A pesar de la corrosión detectada no se apreciaban fisuras longitudinales con la armadura, el saltado del recubrimiento muy local indicaba que el proceso es también solo puntual.

De las figuras se puede deducir que al menos un $50 \%$ de los puntos medidos presentan corrosión activa en el momento de la inspección. Los puntos de medida se han elegido al dividir las diferentes cubiertas en distintos
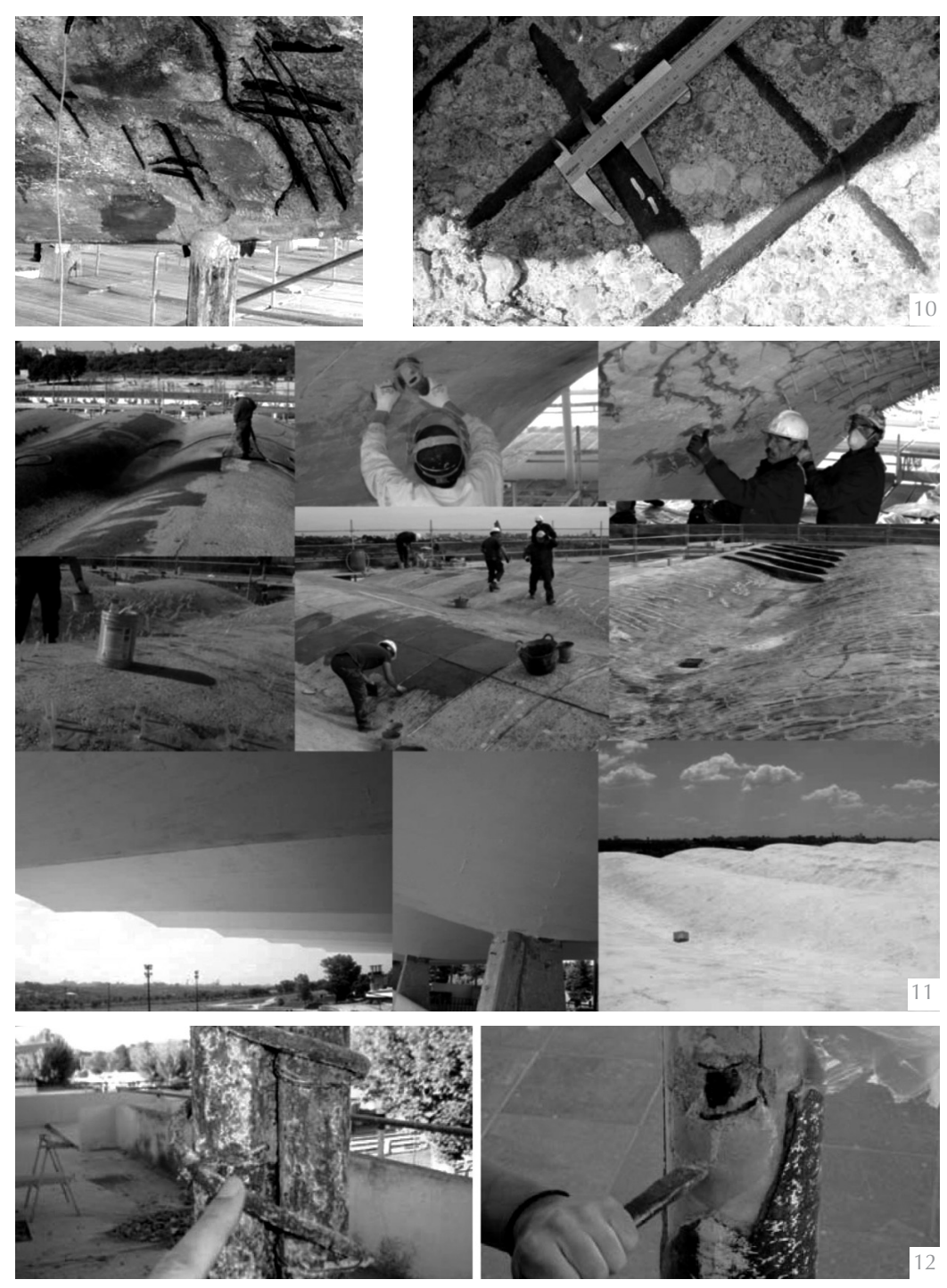

lotes diferenciando la superficie exterior e interior de las cubiertas ( $y$ a su vez las zonas de los valles y senos de cada una de ellas), y los tirantes. Aunque se eligieron puntos que mostraban un cierto deterioro, la corrosión detectada indica que la corrosión esta activa y es necesario acometer alguna actuación para que el proceso no continúe.

\section{INTERVENCIÓN}

En el año 2004, el equipo formado por la oficina Junquera Arquitectos SLP, Carlos Fernández Casado, S.L. y Úrculo Ingenieros, S.L. ganó el concurso internacional que se convocó para restaurar y rehabilitar el Recinto de Carreras. Las obras de rehabilitación realizadas por la empresa DRAGADOS se iniciaron en 2008, siguiendo su curso. En referencia a las láminas de las cubiertas se realizaron las siguientes intervenciones (Figura 11):

1. Eliminación de toda la pintura inferior y la impermeabilización superior mediante chorro de agua.

2. Saneado de las zonas de desprendimiento de material y huecas, limpiando las armaduras vistas, cepillándolas y pasivándolas; para después reparar la zona con un mortero específico.

3. Tratamiento de fisuras mediante su saneo abriéndolas, introduciendo cánulas por las cuales se inyecta resina después de sellarlas con masilla y tapándolas finalmente con mortero de reparación. Este tratamiento se realizó tanto en la superficie superior como en la inferior de las cubiertas.

4. Acabado en la parte inferior con mortero imitando la terminación original con las tablillas de encofrado de madera y posterior pintura anticarbonatación.

5. Impermeabilización de la parte superior con lámina de poliuretano de 3-4 mm. de espesor, sobre mortero de puente de unión de baja retracción.

6. Rehabilitación de los tirantes de las cubiertas. Se retiró el recubrimiento exterior de fibrocemento y el mortero que rodeaba los tirantes metálicos y se saneó hasta llegar a limpiar completamente los mismos (Figura 12).

Después se pintaron con una imprimación impermeable y posteriormente se inyectó una lechada de terminación, estableciendo dos capas para garantizar la impermeabilización.

Las labores de reparación se realizaron de manera satisfactoria y no es objeto de este trabajo su descripción en más detalle.

\section{MONITORIZACION DE LA ESTRUCTURA}

Como se ha mencionado se aprovechó la rehabilitación para colocar sensores que informen sobre la posible aparición de deterioros sin necesidad de extracción de muestras.

\subsection{Ubicación de los grupos de sensores}

Después de la evaluación del estado de corrosión de la estructura se instalaron sensores de corrosión permanentes capaces de indicar 
el riesgo en las cubiertas y en los tirantes. Se instalaron siete grupos de sensores en las tres cubiertas (Figura 13).

\subsection{Descripción de los grupos de sensores}

Cada grupo está compuesto por tres tipos de sensores (Figura 14):

- Sensor de detección de presencia de agua líquida (Doble barrita);

- Sensor de medida del potencial de corrosión $\left(\mathrm{E}_{\text {corr }}\right)$ de la armadura embebida, con electrodo de referencia tipo $\mathrm{Mn} / \mathrm{MnO}$;

- Sensor de medida de la temperatura (Termopar tipo TMC6-HD).

Los tres tipos de sensores instalados son sensores pasivos, lo que significa que no es necesario aplicar ningún estímulo o aporte energético para obtener las correspondientes respuestas. Por ello sólo es necesaria la instalación de un datalogger (se utiliza un $\mathrm{HOBO}$ U12-006) para el registro continuo de los valores.

El sensor de agua líquida es cualitativo ya que informa sobre la presencia/ausencia de agua en estado líquido (condensación) pues desarrolla una diferencia de potencial entre los dos terminales del sensor, Cuando no hay agua en estado líquido, la diferencia de potencial medida entre los dos terminales del sensor es próxima a $0 \mathrm{mV}$, pero cuando existe agua aumenta los valores de diferencias de potencial hasta unos $700 \mathrm{mV}$.

Por otro lado, el sensor de medida de potencial de corrosión lo constituye un electrodo de referencia de $\mathrm{Mn} / \mathrm{MnO}$, que se conecta a la armadura para evaluar su potencial electroquímico. Este parámetro nos indica la probabilidad de que la armadura sufra corrosión.

En cuanto a la temperatura, los termopares utilizados son TMC6-HD, de rango -40 a $50^{\circ} \mathrm{C}$ ) y con resolución de $0,25^{\circ} \mathrm{C}$ a $20^{\circ} \mathrm{C}$

\subsection{Instalación de los grupos de sensores en lass cubiertas}

El procedimiento de instalación de cada uno de los grupos se lleva a cabo en 3 fases: preparación previa de la zona de instalación, la fijación del grupo de sensores y posterior instalación de la caja-estanca de polietileno para el emplazamiento del datalogger.

Preparación previa de la zPara la colocación del grupo de sensores es necesario realizar una pequeña cata en la superficie del hormigón que permita embeber la placa de fijación y distribución de los sensores, para poder conseguir que la capa de impermeabilización que se coloque después sea lo más lisa y continua posible. (Figura 15)
Aparte de la cata en la que se aloja el grupo de sensores, es necesario realizar una roza para poder embeber y llevar el cable para la conexión con la armadura. La conexión con la armadura se asegura con una fijación de soldadura fría para garantizar la continuidad eléctrica.
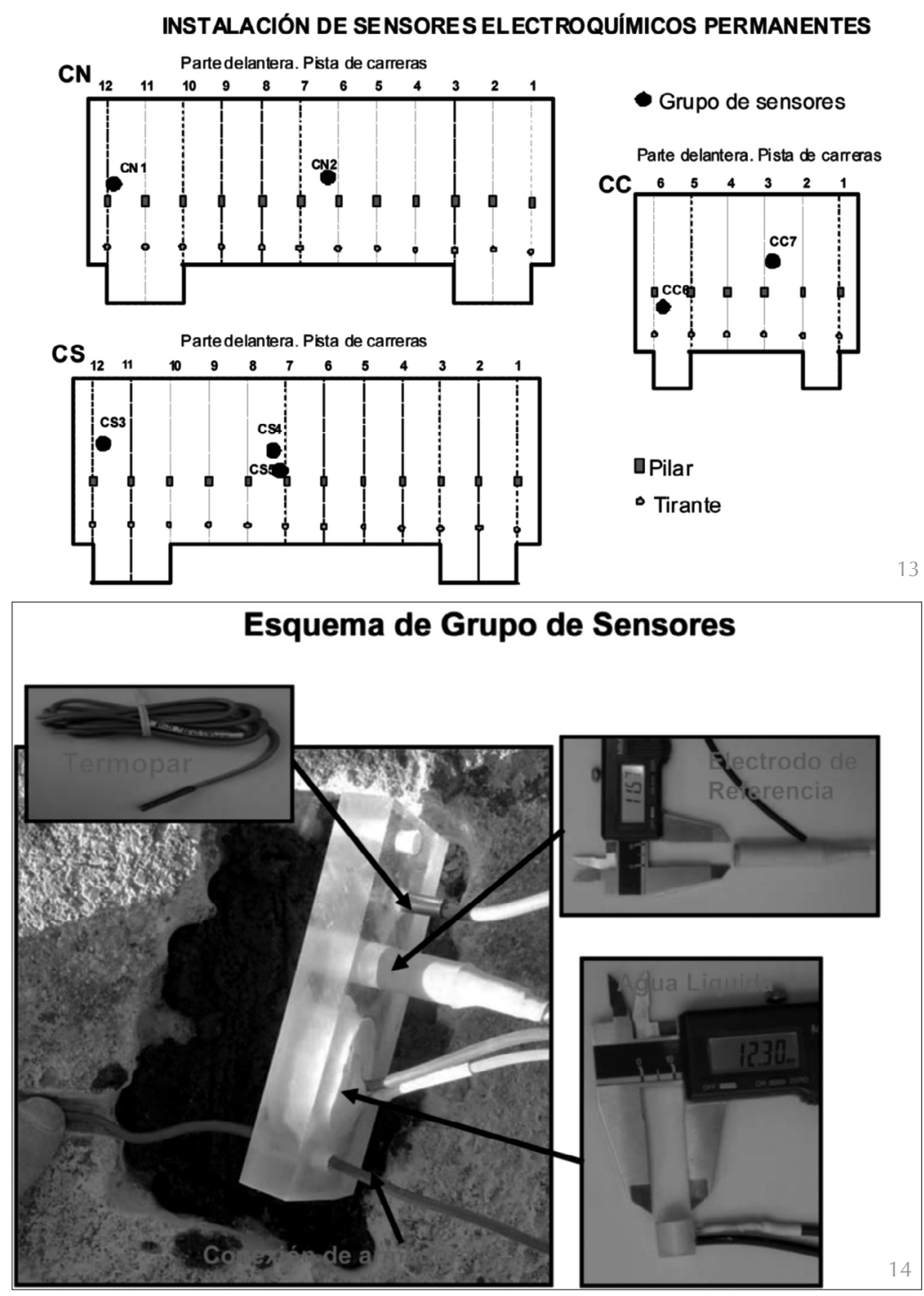

a) Fijación del grupo de sensores.

Una vez realizada la cata donde alojar el grupo de sensores, se prepara un mortero de baja resistividad. Primero se aplica una fina capa de base donde se introducen los diferentes sensores y después se termina rellenando con este mortero toda la cata hasta dejar totalmente embebido el dispositivo. (Figura 16)

b) Instalación de caja de polietileno e impermeabilización.

Una vez instalados los diferentes grupos de sensores en cada una de las cubiertas, se protegieron las terminaciones eléctricas y se aplicó la capa de impermeabilización con lámina de poliuretano de 3-4 mm de espesor a toda la cubierta, poniendo especial
13. Instalación de grupos de senso-

14. Grupo de sensores. res en las cubiertas. 
15. Catas para grupo de sensor.

16. Instalación del grupo de sensores impermeabilización.
17. Zona de sensores. Aplicación de

cuidado en la aplicación en estos lugares. El aspecto de las zonas después de la aplicación de la impermeabilización se puede ver en la Figura 17. Después de la aplicación de la capa de impermeabilización se acopló encima de la zona de las terminaciones eléctricas una cajaestanca de polietileno donde queda instalado el datalogger.

Como ya se ha mencionado, se instalaron en las tres cubiertas un total de 7 grupos de sensores permanentes, de los cuales 6 se instalaron en la superficie superior de las cubiertas Norte, Sur y Central y uno en la parte inferior de la Cubierta Sur. En esta Cubierta Sur se pudieron colocar en la misma zona un grupo por la superficie superior y otro por la parte inferior.
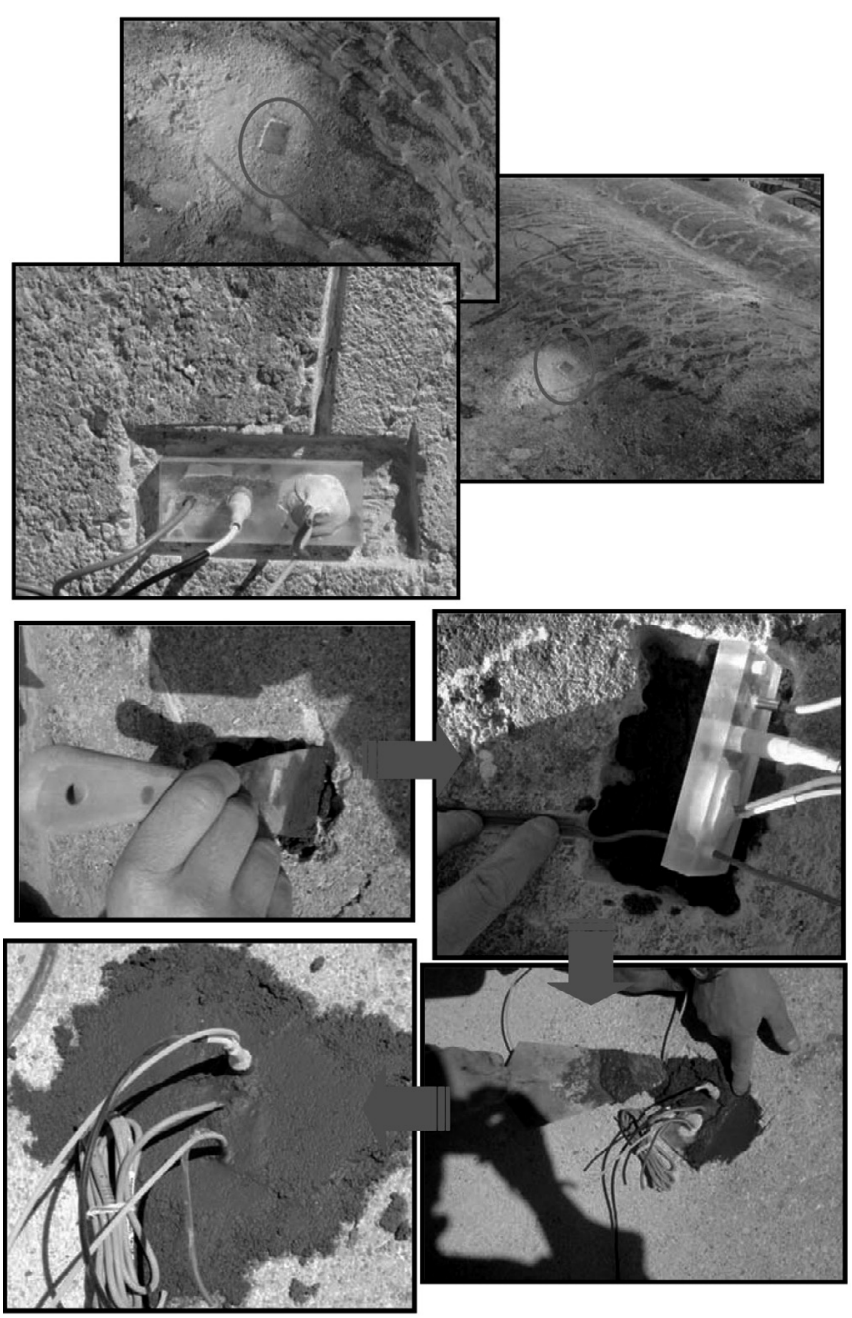

15

16
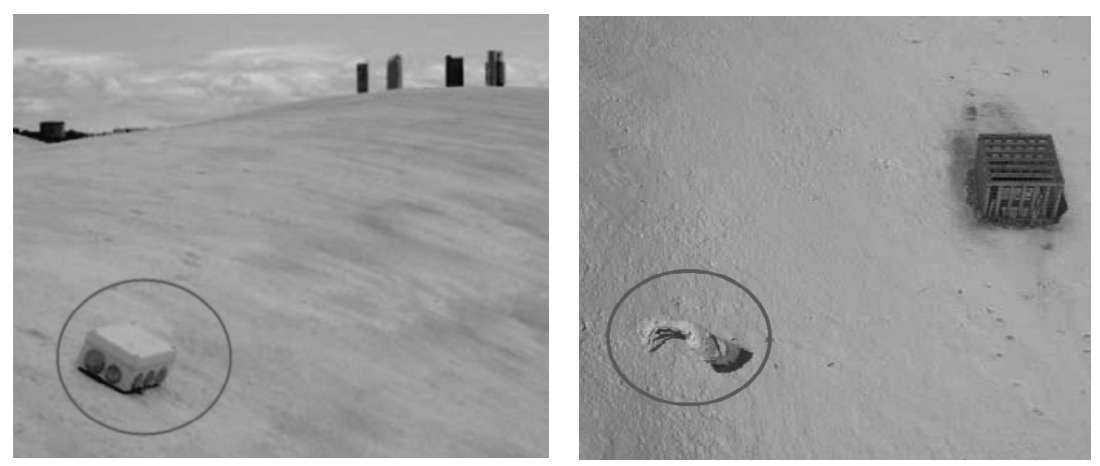

En la Figura 18 se representa la localización de los diferentes grupos en la Cubierta Sur.

\subsection{Resultados de los sensores permanentes}

En la Figura 19 se presentan los valores de potencial de corrosión y de presencia de agua líquida de todos los grupos instalados en las cubiertas.

Se observa cómo la evolución del potencial de corrosión ( $\left.\mathrm{E}_{\text {corr }}\right)$ en prácticamente todos los sensores es hacia valores de potencial más positivos, lo que indica la tendencia a pasivarse de las armaduras tras la reparación. En todos los sensores de agua líquida los valores de diferencia de potencial registrados se sitúan en torno a $20 \mathrm{mV}$, lo que indica la no presencia de agua líquida en el interior del hormigón.

\section{CONCLUSIONES}

Las cubiertas del Hipódromo, que siguen asombrando por su ligereza y esbeltez, han resistido el paso del tiempo y la acción de los agentes atmosféricos de una forma sobresaliente, al presentar solo deterioros por corrosión de las armaduras muy locales por fallos en el sistema de impermeabilización que se debió aplicar en los años 60. Del trabajo actual desarrollado para su rehabilitación integral se pueden expresar de manera resumida y esquemática las siguientes conclusiones más relevantes relativas a los trabajos para el estudio de su durabilidad y control permanente de los parámetros de corrosión:

- Antes de la intervención la estructura presentaba un grado de carbonatación alto en la parte inferior de todas las cubiertas. Aun no habiendo grandes pérdidas de sección de manera general, toda la armadura inferior presentaba corrosión generalizada, cuantificada mediante técnicas electroquímicas no destructivas.

- La corrosión que se detectó solo en las zonas donde se había deteriorado la impermeabilización, es mayor en la superficie superior de la cubierta por el acceso del agua de lluvia en las zonas donde la lámina asfáltica estaba deteriorada. Esta impermeabilización ha resultado esencial,

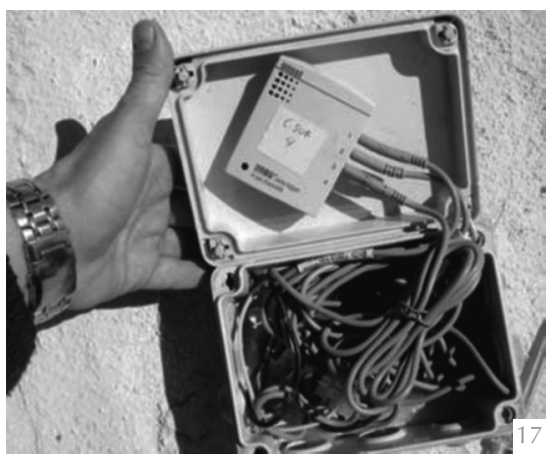


ya que solo satura el hormigón a grados que induce la corrosión activa la acción directa del agua de la lluvia. Las zonas inferiores de las cubiertas, aunque carbonatadas, no han llegado a un grado de corrosión significativo.

- En cuanto a las tres barras asimétricas que componen cada uno de los tirantes, esenciales en el comportamiento estructural, pese al peculiar mecanismo de impermeabilización que se encontró compuesto por una camisa tubular de fibrocemento, su estado tras 75 años de exposición al ambiente era muy aceptable, presentando escasa carbonatación en su recubrimiento de mortero y ausencia de corrosión.

- Después de la intervención los resultados después de un año procedente de los sensores permanentes embebidos, muestran que la reparación de las cubiertas se ha ejecutado de forma satisfactoria, ya que los potenciales de la armadura presentan valores de ausencia de riesgo de corrosión al igual que los sensores de agua indican la ausencia de condensación.

- Es importante resaltar la importancia de cuantificar las velocidades de corrosión para la predicción de la evolución futura del deterioro, así como la ayuda que suponen los sensores instalados de forma permanente para el control no destructivo de la eficacia de la reparación.

\section{AGRADECIMIENTOS}

Los autores expresan su agradecimiento a la empresa propietaria del Hipódromo de la Zarzuela y a DRAGADOS que efectúo la rehabilitación por su colaboración y apoyo. Además se quería agradecer la participación tanto económica como personal del proyecto de investigación del Ministerio de Ciencia e Innovación, SEDURECCONSOLIDER-INGENIO 2010.

\section{BIBLIOGRAFÍA}

(1) Torroja, E.: "Estructura de la Tribuna del nuevo Hipódromo de Madrid". Revista de Obras Públicas, junio 1941

(2) Torroja, E.: "Madrid Racehouse". Architectural Record, VI, 1958

(3) "Hipódromo de la Zarzuela". Informes de la Construcción, 154-6, 1962.

(4) Andrade, C.; Martínez, I.; Castellote, M.; Zuloaga, P.: "Some principles of service life calculation of reinforcements and in situ corrosion monitoring by sensors in the radioactive waste containers of $\mathrm{El}$ Cabril disposal (Spain)". Journal of Nuclear Materials 358 (2006) 82-95.

(5) Martínez, I.; Castillo, A.; Andrade, C.: "Non destructive electrochemical techniques applied to the corrosion evaluation of the liner structures in nuclear power plants". Journal of Nuclear Materials. ELSEVIER. Volumen 373 (2008) 226-236.

(6) Feliú, S.; González, J. A.; Feliú, S. Jr.; Andrade, C.: "Confinement of the electrical signal or in-situ measurement of Polarization Resistance in Reinforced concrete", ACI Mater. J., 87, pp. 457 (1990).

(7) Andrade, C.; Alonso, M. C.; Gonzalez, J. A.: "Corrosion Rates of Steel in Concrete", ASTM STP, vol. 1065, American Society for Testing and Materials, Philadelphia, 1990, p. 29.

(8) ASTM C-876-91, ASTM, vol. 04.02, 1995

(9) UNE 112-0111-94: "Corrosión de armaduras. Determinación de la profundidad de carbonatación en hormigones endurecidos y puestos en servicio".

(10) Andrade, C.; Alonso, C.; Gulikers, J.; Polder, R.; Cigna, R.; Vennesland, O. and Salta, M.: "Test methods for on-site reinforcement corrosion rate measurement of steel reinforcement in concrete by means of the Polarization Resistance method". RILEM Recommendation of TC-154 "Electrochemical Techniques for measuring metallic corrosion". Materials and Structures, vol. 37, nov. 2004, pp. 623.643.
18. Grupos de sensores en la Cubierta Sur.

19. Medidas de los sensores instalados. 